\title{
RINGS WITH QUASI-CONTINUOUS RIGHT IDEALS
}

\author{
S. K. JAIN, S. R. LÓPEZ-PERMOUTH and S. RAZA SYED \\ Ohio University, Athens, Ohio 45701, USA
}

(Received 21 May, 1997)

\begin{abstract}
Rings in which each right ideal is quasi-continuous (right $\pi$-rings) are shown to be a direct sum of semisimple artinian square full ring and a right square free ring. Among other results it is also shown that (i) a nonlocal right continuous indecomposable right $\pi$-ring is either simple artinian or a ring of matrices of a certain type, and (ii) an indecomposable non-local right continuous ring is both a right and a left $\pi$-ring if and only if it is a right q-ring. In particular, a non local indecomposable right q-ring is a left q-ring.
\end{abstract}

0. Introduction. Rings for which every right ideal is quasi-injective (known as right $q$-rings) have been studied by several authors (c.f. [4], [5], [6], [7]). The purpose of our paper is to extend this line of research by studying rings in which every right ideal is quasi-continuous (right $\pi$-rings). In Section 2 we show that a $\pi$-ring is a direct sum of a semisimple artinian square full ring and a square free ring.

In Section 3 we study right continuous right $\pi$-rings. We show that a non-local, right continuous, indecomposable right $\pi$-ring $R$ is either simple artinian or a ring of matrices of a certain type (Theorem 3.8). We show that an indecomposable, nonlocal, right continuous ring $R$ is both a right and a left $\pi$-ring if and only if $R$ is a right $q$-ring (Theorem 3.13). In particular, under these hypotheses, $R$ is a right $q$-ring if and only if it is a left $q$-ring.

1. Definitions and preliminaries. Throughout the paper $R$ will be a ring with identity and all $R$-modules will be unital right $R$-modules, unless otherwise stated. For modules $M, N$, the notations $N \subset^{\prime} M$ and $N \subset^{\oplus} M$ respectively serve to denote that $N$ is an essential submodule of $M$ and that $N$ is a direct summand of $M$. By $Z(M)$, $\operatorname{Soc}(M)$ we denote the singular submodule and socle of $M$, respectively. $J(R)$ is the Jacobson Radical of $R$. $\hat{M}$ (or $E(M)$ ) stands for the injective hull of $M$. $\operatorname{Hom}_{R}(M, N)$ stands for the set of $R$-homomorphisms from $M$ to $N$. $\operatorname{End}_{R}(M)$ is the set of $R$-endomorphisms of $M$. If $N \subset M$, a closure of $N$ in $M$ is a maximal essential extension of $N$ in $M . N$ is said to be closed in $M$ if $N$ has no proper essential extension on $M$. Given right $R$-modules $M$ and $N, M$ is said to be $N$-injective iff for any $K \subset N$, every $\alpha \in \operatorname{Hom}_{R}(K, M)$ is the restriction of some $\beta \in \operatorname{Hom}_{R}(N, M)$.

For a module $M$ we consider the following conditions.

$\left(C_{1}\right)$ For every submodule $N$ of $M$ there exists a summand $L$ of $M$ with $N \subset^{\prime} L$.

$\left(C_{2}\right)$ If a submodule $N$ of $M$ is isomorphic to a summand of $M$ then $N$ itself is a summand of $M$.

$\left(C_{3}\right)$ If $A$ and $B$ are summands of $M$ with $A \cap B=0$ the $A \oplus B$ is a summand of $M$. 
A module $M$ is called (quasi-)continuous if it satisfies $\left(C_{1}\right)$ and $\left(C_{2}\right)\left(\left(C_{1}\right)\right.$ and $\left(C_{3}\right)$ ). A module $M$ is called a $C S$ (or extending) module if it satisfies $\left(C_{1}\right)$. Equivalently, $M$ is a $C S$ module if every closed submodule of $M$ is a direct summand of $M$.

It is well known that a module $M$ is quasi-continuous if and only if $e M \subset M$ for every idempotent $e \in \operatorname{End}(\hat{M})$ if and only if every decomposition $E(M)=\bigoplus_{i \in I} E_{i}$ induces $M=\oplus_{i \in I}\left(M \cap E_{i}\right)[10,2.8]$.

Definition 1.1. A ring $R$ is said to be a right $(f) \pi$-ring if every (finitely generated) right ideal in $R$ is quasi-continuous.

REMARK 1.2. A right uniform ring is a right $(f) \pi$-ring.

The following Lemma is a particular case of [9, Proposition 2.6.]. This may already be known but we have not found it anywhere in the literature.

Lemma 1.3. A right $R$-module $M$ is quasi-continuous if and only if $M$ satisfies condition $\left(C_{1}\right)$ and, whenever $M=A \oplus B, A$ and $B$ are mutually injective.

Proof. The 'only if' part is well known (see Lemma 1.7 below). For completeness, we include here the proof of the other implication.

Let $\hat{M}=E_{1} \oplus E_{2}$. We want to show that $M=A \oplus B$ where $A=E_{1} \cap M$ and $B=E_{2} \cap M$. Since $A$ is closed in $M$ and $M$ satisfies $\left(C_{1}\right), M=A \oplus X$ for some $X \subset M$. Let $\pi_{A}$ and $\pi_{X}$ be the corresponding projection maps. Then $B \cong \pi_{X}(B) \subset X$. Define $\alpha: \pi_{X}(B) \rightarrow A$ by $\alpha\left(\pi_{X}(b)\right)=\pi_{A}(b)$. The map $\alpha$ is well-defined since $\pi_{X}: B \rightarrow \pi_{X}(B)$ is an isomorphism. But $A$ is $X$-injective, so $\alpha$ extends to $\beta: X \rightarrow A$. Let $X^{*}=\{x+\beta(x) \mid x \in X\}$. Then $M=A \oplus X^{*}$. Also $B \subset^{\prime} X^{*}$ and $B$ is closed. This implies that $B=X^{*}$ and, consequently, $M=A \oplus B$.

Lemma 1.3 has the following immediate consequence, an intrinsic characterization of $(f) \pi$-rings:

THEOREM 1.4. A right ring $R$ is a right $(f) \pi$-ring if and only if every (finitely generated) right ideal of $R$ satisfies $\left(C_{1}\right)$ and, whenever $A$ and $B$ are right ideals of $R$ with $A \cap B=0, A$ and $B$ are mutually injective.

Proof. Immediate from Lemma 1.3.

We list below some well known results that will be used frequently.

Theorem 1.4 may be strengthened by observing that in order to check if a ring $R$ is right $(f) \pi$, it suffices to concentrate on the essential right ideals.

THEOREM 1.5. For a ring $R$ the following conditions are equivalent:

(1) $R$ is a right $(f) \pi$-ring,

(2) every essential (finitely generated) right ideal of $R$ is quasi-continuous,

(3) every essential (finitely generated) right ideal satisfies $\left(C_{1}\right)$ and, whenever $A$ and $B$ are right ideals such that $A \oplus B \subset^{\prime} R, A$ and $B$ are mutually injective. 
Proof. It follows from the above characterizations of $(f) \pi$-rings since, when $R$ satisfies $\left(C_{1}\right)$, every (finitely generated) right ideal is a direct summand of a (finitely generated) essential right ideal.

Lemma 1.6. Let $M$ and $N$ be two right R-modules. Then $M$ is $N$-injective iff for any $\alpha \in \operatorname{Hom}_{R}(N, \hat{M}), \operatorname{Im} \alpha \subset M$.

Proof. See $[\mathbf{1 0}, 1.13]$.

Lemma 1.7. If $M=\oplus_{i=1}^{n} M_{i}$ then $M$ is quasi-continuous iff each $M_{i}$ is quasi-continuous and $M_{i}$ is $M_{j}$-injective for all $i \neq j$.

Proof. See [10, 2.14].

Lemma 1.8. A right quasi-continuous ring $R$ is right continuous iff $J(R)=Z(R)$ and $R / J(R)$ is a regular ring.

Proof. See [10, 3.15].

LEMMA 1.9. In a right quasi-continuous ring the idempotents modulo $Z(R)$ can be lifted.

Proof. See [10, 3.7].

A right module $M$ is called local if $M$ contains a unique maximal submodule. A ring $R$ is called local ring if the right $R$-module $R_{R}$ is local. Let $e$ be an idempotent in $R$. Then $e$ is called primitive if the right ideal $e R$ is indecomposable. Furthermore, $e$ is called local if the ring $\operatorname{End}_{R}(e R)$ is local or equivalently if $e J$ is the unique maximal submodule of $e R[\mathbf{8}, 21.18]$.

Lemma 1.10. A primitive idempotent in a right continuous ring is local.

Proof. Let $e$ be a primitive idempotent in a continuous ring $R$. Then $e R e$ is a continuous ring [10, 3.8] and contains no nontrivial idempotents [8, 21.8]. By Lemmas 1.7 and 1.8, eRe/eJe is a division ring. It follows that eJe is the maximal right ideal in $e R e$ and, therefore, $e$ is local $[8,21.9]$.

Local rings have nontrivial idempotents. We see next that the converse also holds when the ring is continuous.

Lemma 1.11. A right continuous ring $R$ is local iff it contains no nontrivial idempotents.

Proof. Let $R$ be a right continuous ring with no nontrivial idempotents. Then $R / J(R)$ is a regular ring with no nontrivial idempotents. Hence $R / J(R)$ is a division ring. It follows that $R$ is a local ring. The converse is trivial.

Two modules $M$ and $N$ are said to be orthogonal if no submodule of $M$ is isomorphic to a submodule of $N$. Let $M, X$ be arbitrary right $R$-modules and $N$ a submodule of $M$. Then $M$ is said to be a square if $M \cong X^{2}$. The $N$ is called a square 
root in $M$ if $N^{2}$ is embeddable in $M . M$ is called square free if $M$ contains no square roots. $M$ is called square full if every submodule of $M$ contains a square root in $M$.

2. Rings with quasi-continuous right ideals. Right $(f) q$-rings may be characterized as those right self-injective rings for which every (finitely generated) essential right ideal is a two sided ideal [7]. For right $(f) \pi$-rings one obtains the following.

Proposition 2.1. A ring $R$ is a right $(f) \pi$-ring if and only if $R$ is quasi-continuous and every (finitely generated) essential right ideal is a left $S$-module, where $S$ is the subring of $R$ generated by its idempotent elements.

Proof. From Theorem 1.5, in order to check to see if $R$ is an $(f) \pi$-ring, it suffices to check that every (finitely generated) essential right ideal in $R$ is quasi-continuous. We'll show here that a (finitely generated) essential right ideal $I$ of a quasi-continuous ring $R$ is quasi-continuous if and only if it is a left $S$-module. Our result will then follow. Let $R$ be a quasi-continuous ring. The subring $T$ generated by all the idempotents of the $\operatorname{ring} \operatorname{End}_{R}(\hat{R})$ can then be viewed as the subring $S$ generated by all the idempotents of the ring $R$. Let $I$ be a (finitely generated) essential right ideal of $R$. Then, $I=T I=S I$ if and only if $I$ is quasi-continuous.

Lemma 2.2. Let $A, B$ be right ideals in a right f $\pi$-ring $R$ with $A \cap B=0$ and $A \cong B$. Then

(a) every finitely generated right ideal in $A$ or $B$ is injective. Moreover, if $R$ is a right $\pi$-ring then $A$ and $B$ are semisimple and injective, and

(b) the right ideals $A$ and $B$ are nonsingular.

Proof. For the first part (a) we can assume that $A$ and $B$ themselves are finitely generated. By the quasi-continuity of $R$, there exists an idempotent $e^{2}=e \in R$ such that $A \subset^{\prime} e R \subset^{\oplus} R$. The fact that $B \cap e R=0$ implies that $B$ is $e R$-injective. And $A \cong B \Rightarrow A$ is $e R$-injective. But $A$ is also $(1-e) R$-injective, therefore $A$ is $R$-injective. For the second part of (a), assume that $R$ is a $\pi$-ring and $0 \neq X \subset A$ then $X \cong \alpha(X)$ where $\alpha: A \rightarrow B$ is a given isomorphism. By a similar argument as above $X$ is injective. Thus, $A$ is semisimple and so is $B$. Let us now consider (b). Let $x$ be an element of $Z(A)$. Since $x R$ is injective, there exists an idempotent $e \in R$ such that $e R=x R<Z(A)$. Since $Z(R)$ contains no nonzero idempotents, it follows that $x=0$.

Lemma 2.3. Let $A, B$ be right ideals in a ring $R$ with $A \cap B=0$. Let $\alpha: A \rightarrow B$ be a nonzero homomorphism.

(a) If $R$ is a right $\pi$-ring then the image of $\alpha$ is semisimple.

(b) If $R$ is a right f $\pi$-ring and $B$ is uniform then the image of $\alpha$ is simple.

(c) Let $R$ be a right fл-ring with a non-trivial primitive idempotent e such that $e R(1-e) \neq 0$. Then $e R$ contains a simple right ideal.

Proof. (a) Let $L \subset^{\prime} B$. Since $L \oplus A$ is quasi-continuous, and $\alpha$ may be viewed as $\alpha: A \rightarrow \hat{L}, \operatorname{Im} \alpha \subset L$. It follows that $\operatorname{Im} \alpha \subset \operatorname{Soc}(B)$.

(b) Let $a$ and $x$ be a nonzero elements in $A$ and $B$ respectively. Then $\alpha: A \rightarrow B \subset \hat{B}=\widehat{x R}$ implies $\alpha(a R) \subset x R$ since $a R \oplus x R$ is quasi-continuous. It follows that $\operatorname{Im} \alpha \subset x R$. Then $0 \neq \operatorname{Im} \alpha \subset \operatorname{Soc}(B)$, a simple right $R$-module. 
(c) Since $e R$ quasi-continuous and indecomposable, $e R$ is uniform. Thus, the result follows from (b).

Recall that a homogeneous component of an $R$-module is a complete sum of mutually isomorphic simple submodules.

LEMma 2.4. For a right $\pi$-ring $R$, we have the following.

(1) Let $A, B$ be independent right ideals of $R$ with $B$ an epimorphic image of $A$. Then $B$ is cyclic.

(2) Let $\{A\} \cup\left\{B_{i}: i \in I\right\}$ be an independent family of right ideals in $R$ with $\oplus_{i \in I} B_{i}$ an epimorphic image of $A$. Then the index set $I$ is finite.

Proof. Let $\alpha$ be a homomorphism from $A$ onto $B$. There exists an idempotent $e$ in $R$ such that $e R$ is a closure of $A$ in $R$. As $R$ is a $\pi$-ring, $\alpha$ extends to an epimorphism from $e R$ onto $B$. Hence $B$ is cyclic and the proof of (1) follows. The proof of (2) is immediate from (1).

TheOREM 2.5. A right $\pi$-ring $R$ has only finitely many nonsimple homogeneous components and each one of them is injective.

Proof. Let $\left\{H_{i}: i \in I\right\}$ be the family of homogeneous components of a right $\pi$ ring $R$. For each $i \in I$, let $S_{i}$ and $T_{i}$ be minimal right ideals such that $H_{i}=\left[S_{i}\right]=\left[T_{i}\right]$ and $S_{i} \cap T_{i}=0$. Then there exists an isomorphism $\oplus_{I \in i} S_{i} \rightarrow \oplus_{i \in I} T_{i}$. By Lemma $2.4(2),|I|<\infty$. Hence $R$ has only finitely many non-simple homogeneous components. Now let $H$ be a non-simple homogeneous component of $R$. If $H$ is not finitely generated then there exist independent submodules $A, B$ of $H$ such that $A \cong H \cong B$. By Lemma 2.2, $A, B$ are injective. But then $H$ is finitely generated, a contradiction. So, $H$ is finitely generated.

THEOREm 2.6. A right $\pi$-ring is a direct sum of a square full semisimple artinian ring and a right square free ring.

Proof. Let $R$ be a right $\pi$-ring and $H$ the direct sum of all square full homogeneous components of $R$. By Lemma 2.3, each simple submodule of $H$ is injective, and by Theorem 2.5, $H$ is injective. Let $H=e R$ for some idempotent $e \in R$. Since $e R$ and $(1-e) R$ are orthogonal, it follows that $(1-e) R e=0=e R(1-e)$. Hence the decomposition $R=e R \oplus(1-e) R$ is a ring decomposition. Now, suppose $(1-e) R$ contains a square. Then there exist right ideals $A, B$ in $(1-e) R$ with $A \cap B=0$ and $A \cong B$. By Lemma $2.3, A, B$ are semisimple. It follows that $(1-e) R$ contains a square full homogeneous component, a contradiction. Hence, $(1-e) R$ is square free and we get the desired decomposition.

COROllary 2.7. An indecomposable ring $R$ containing a square is a right $\pi$-ring iff $R$ is simple Artinian.

Proposition 2.8. Let $R$ be a right $\pi$-ring. Suppose $\left\{A_{i}\right\}_{i \in I}$ is an independent family of right ideals in $R$. If for each $i \in I$ there exists a right ideal $B_{i}$ in $R$ that is a homomorphic image of $A_{i}$ with $A_{i} \cap B_{i}=0$ then I is finite. 
Proof. Suppose $I$ is infinite. In view of Theorem 2.6 we can assume that $R$ is square free. By Lemma 2.3 we can assume the $B_{i}$ are simple. Since $R$ is a $\pi$-ring, any homomorphism from $A_{i}$ onto $B_{i}$ can be extended to a homomorphism from $e_{i} R$ to $B_{i}$, where $e_{i} R$ is a closure of $A_{i}$ in $R$ and $e_{i}$ is an idempotent in $R$. As $R$ is square free, using the projectivity of $e_{i} R$ one can assume that $B_{i}$ are independent. Pick an $A_{i_{1}}$. By Lemma 2.4, images of the $A_{i}$ are contained in $A_{i_{1}}$ for only finitely many $i$. Pick an $A_{i_{2}}$ whose image is not contained in $A_{i_{1}}$. Again by Lemma 2.4, images of the $A_{i}$ are contained in $A_{i_{2}}$ for only finitely many $i$. Hence there exists an $A_{i_{3}}$ whose image is not contained in $A_{i_{1}} \oplus A_{i_{2}}$. Clearly this process is inductive.

Hence there exists an infinite subset $I^{\prime} \subset I$ such that $\left(\oplus_{i \in I^{\prime}} A_{i}\right) \cap\left(\oplus_{i \in I^{\prime}} B_{i}\right)=0$. Now for each $i \in I^{\prime}$, the homomorphisms from $A_{i}$ onto $B_{i}$ induce an epimomorphism from $\oplus_{i \in I^{\prime}} A_{i}$ onto $\bigoplus_{i \in I^{\prime}} B_{i}$. By Lemma 2.4, $I^{\prime}$ is finite, a contradiction. Hence the proof follows.

3. Continuous $(\boldsymbol{f}) \boldsymbol{\pi}$-rings. In this section we will study the $(f) \pi$-rings that are continuous. Since, by Lemma 1.9, a continuous ring is local if and only if it contains no non-trivial idempotents, a continuous, local $(f) \pi$-ring is uniform. Conversely, all right uniform rings are $\pi$-rings (Remark 1.2.). Consequently, from now on, we shall only consider non-local rings. We completely characterize indecomposable, nonlocal, right $\pi$-rings that are right continuous. We prove that such rings are either simple artinian or a certain type of rings of matrices.

Lemma 3.1. Let $R$ be a right continuous right f $\pi$-ring, e a primitive idempotent in $R$ and $\alpha: e R \rightarrow R$ a nonzero homomorphism with $\operatorname{Im} \alpha \cap e R=0$. Then

(a) $\operatorname{Im} \alpha$ is simple;

(b) $e R(1-e) \neq 0$.

In particular, for a right continuous, right f $\pi$-ring $R$ and primitive idempotent $e \in R$, if $(1-e) R e \neq 0$ then $e R(1-e) \neq 0$.

Proof. (a). Let $\alpha: e R \rightarrow R$. By Lemma 1.8, $e R$ is local and therefore $\alpha(e R)$ is local. It follows that $\alpha(e R)$ is indecomposable and therefore uniform $(\alpha(e) R$, being finitely generated, is quasi-continuous). Thus, by Lemma 2.3(b), Im $\alpha$ is simple.

(b). Let $\operatorname{Im} \alpha \subset^{\prime} f R$. Then, $f R \cap e R=0$ and, therefore, $R=(f R \oplus e R) \oplus L$ for some $L \subset R$, since $R$ is quasi-continuous. Thus, $(1-e) R \cong \frac{R}{e R} \cong f R \oplus L$ and, therefore, $A$ is embedded in $(1-e) R$. Hence we can assume that $\alpha: e R \rightarrow(1-e) R$.

Assume $e R(1-e)=0$. By Lemma $1.8, e R e=e R$ is local ring with its unique maximal ideal $e J$ where $J=J(R)$. If $e J=0, e R$ is simple right $R$-module and $\alpha(e R) \cong e R$. The inverse of $\alpha$ from $e R$ into $\alpha(e R)$ extends to some nonzero homomorphism from $(1-e) R$ to $e R$, proving our claim. Let $x$ be a nonzero element in $e J$. Since $e R e$ is local there exists an $e$ Re-epimorphism $\beta: x e R \rightarrow \frac{e R}{e J}$. Furthermore, since $e R=e R e, \beta$ is an $R$-homomorphism as well.

Since $\operatorname{Im} \alpha$ is simple (by (a)), $\frac{e R}{e J} \cong \operatorname{Im} \alpha \subset(1-e) R$. Let $\gamma$ be a nonzero homomorphism from $\frac{e R}{e J}$ to $(1-e) R$. Then $\gamma \beta$ is a nonzero $R$-homomorphism from $x e R$ to $(1-e) R$. Since $R$ is quasi-continuous, $\gamma \beta$ extends to a nonzero $R$-homomorphism, $\delta$ say, from $e R$ to $(1-e) R$. But $x e R \subset e J=\operatorname{Ker} \delta$ (by (a)). This implies that 
$0=\delta(x e R)=\gamma \beta(x e R)=\operatorname{Im} \alpha$, a contradiction since $\alpha \neq 0$. Thus $e R(1-e) \neq 0$ and the proof follows.

LEMma 3.2. Let $R$ be a continuous square free f $\pi$-ring with a primitive idempotent e such that $e R(1-e) \neq 0$. Then eRe is a division ring and $e R(1-e)$ is the only proper submodule of $e R$.

Proof. By Lemma $2.3 S=\operatorname{Soc}(e R)$ is non-zero. Since $e R$ is continuous, $S$ is simple. By Lemma 2.3, we get $e R(1-e) \subseteq S$. Now let $0 \neq s \in S$ and $0 \neq \alpha:(1-e) R \rightarrow e R$. Clearly, by Lemma $2.3, \operatorname{Im} \alpha=S$. If $s e \neq 0$ then $S=s e R$ is a homomorphic image of $e R$ under some $\beta: R \rightarrow s R$ given by $\beta(e x)=\operatorname{sex}$. By the projectivity of $e R$ there exists a homomorphism $\gamma: e R \rightarrow(1-e) R$ so that $\alpha \gamma=\beta$. By Lemma 3.1, $\operatorname{Im} \gamma$ is simple. It follows that $\operatorname{Im} \gamma \cong S$, a contradiction since $R$ is square free. Hence $s e=0$ and $s \in e R(1-e)$. Consequently, $S=e R(1-e)$. Now, let $J=J(R)$. Then eJe is the Jacobson radical of $e R e$. Since $R$ is continuous $J(R)=Z(R)$ and therefore $J S=0$. Hence $(e J e) S=(e J)(e S)=e J S=0$. Thus, $e J e[e R(1-e)]=0$, and so eJeR $=e J e R e \subseteq e J e$. Hence eJe is an $R$-submodule of $e R$. As $S=e R(1-e)$ it follows that $(e J e) \cap S=0$. But $e R$ is uniform, so eJe $=0$. Thus $e R e$ is a division ring. Now let $I \subseteq e R$. Then $S \subseteq I$ (since $e R$ is uniform). It follows that $S \subseteq I(1-e) \subseteq e R(1-e)=S$. Hence $I(1-e)=S$. Furthermore, since $I e$ is an $e R e$-submodule of $e R e$, it follows that either $I e=0$ or $I e=e R e$. If $I e=0$ then $I=I(1-e)=S$. If $I e=e R e$ then, since $I=I e \oplus I(1-e)$, it follows that $I=e R e \oplus e R(1-e)=e R$. Hence $S$ is the only proper submodule of $e R$.

THeOREM 3.3. Let $R$ be an indecomposable, non-local, right $\pi$-ring. If $R$ is right continuous then $R$ has essential socle.

Proof. If $R$ contains a square then, by Corollary $2.7, R$ is simple artinian and we are done. Assume $R$ is square free. Since $R$ is non-local and indecomposable it contains a nontrivial idempotent $f$ and either $f R(1-f)$ or $(1-f) R f$ is nonzero (by Lemma 1.9). Therefore, by Lemma $2.3, R$ has nonzero socle. Let $e^{2}=e \in R$ with $\operatorname{Soc}(R) \subset^{\prime} e R$. Suppose $e \neq 1$. For any non-zero idempotent $g \in(1-e) R$, since $\operatorname{Soc}(g R)=0,(1-g) R g \neq 0$ and $g$ is not primitive (Lemmas 2.3, 3.1). Indeed, for any $\alpha: g R \rightarrow(1-g) R$, Im $\alpha$ is semisimple. So $a: g R \rightarrow e R$. Furthermore, for any two non-zero orthogonal idempotents $g_{1}, g_{2} \in(1-e) R$ and non-zero $\alpha_{i}: g_{i} R \rightarrow e R,(i=$ $1,2)$, one gets that $\operatorname{Im} \alpha_{1} \cap \operatorname{Im} \alpha_{2}=0$, for, otherwise, there exists a minimal right ideal $S \subset e R$ such that $g_{1} R$ and $g_{2} R$ map onto $S$. But then by the projectivity of $g_{1} R$ there exists a non-zero $\phi: g_{1} R \rightarrow g_{2} R$. By Lemma 2.3, $\operatorname{Im} \phi$ is semisimple, a contradiction.

Let $1-e=f_{1}+g_{1}$, a sum of orthogonal non-zero idempotents and $0 \neq \alpha_{1}: g_{1} R \rightarrow e R$. Write $f_{1}=f_{2}+g_{2}$, a sum of non-zero orthogonal idempotents and $0 \neq \alpha_{2}: g_{2} R \rightarrow e R$. Continue like this writing $f_{i}=f_{i+1}+g_{i+1}$ and considering $0 \neq \alpha_{i+1}: g_{i+1} R \rightarrow e R$. Then the sum of $\operatorname{Im} \alpha_{i}$ is direct and there exists an epimorphism $\alpha: \oplus_{i=1}^{\infty} g_{i} R \rightarrow \bigoplus_{i=1}^{\infty} \operatorname{Im} \alpha_{i}$. This is a contradiction in view of Lemma 2.4. Therefore $e=1$, proving that $\operatorname{Soc}(R) \subset^{\prime} R$.

Lemma 3.4. Let $R$ be a continuous ring. Suppose e is a nonzero idempotent of $R$. If, for $a, b \in R, a R, b R$ are two non-isomorphic minimal right ideals of $R$ that are homomorphic images of $e R$, then there exists a nonzero idempotent $f$ in $e R \cap a^{\perp}$ such that $f \notin b^{\perp}$, where $x^{\perp}$ is the right of $x$ in $R$. 
Proof. As $a R$ and $b R$ are non-isomorphic, there exists a nonzero element $x$ in $e R \cap a^{\perp}$ but not in $e R \cap b^{\perp}$. Let $\bar{R}=R / J(R)$. Then $\overline{e R e}=e R e / e J e$ is regular [10, 3.11]. Hence there exists $\bar{y} \in \overline{e R e}$ such that $\overline{x y}$ is a nonzero idempotent in $\overline{e R e}$. Thus there exists a nonzero idempotent $f$ in $e$ Re such that $f-x y \in e J e$ (Lemmas 1.8, 1.9). Thus $f \in e R \cap a^{\perp}$. Now, if $f \in b^{\perp}$, then $x y \in b^{\perp}$. But, as $x-x y x \in b^{\perp}$, it follows that $x \in b^{\perp}$, a contradiction. Hence, $f \notin b^{\perp}$.

THeOREM 3.5. Let $R$ be a continuous $\pi$-ring. For any two independent right ideals $A$ and $B$ in $R, A$ has only finitely many simple images in $B$.

Proof. Let $I$ be an infinite index set such that for each $i \in I, S_{i}$ is a simple image of $A$ in $B$. For $e^{2}=e \in R$, let $e R$ be a closure of $A$ in $R$. As $R$ is a $\pi$-ring, each $S_{i}$ is an image of $e R$. Let $S_{i}=a_{1} R$ for some $a_{i} \in R$. For $i \neq j, i, j \in I$, there exists, by Lemma 3.4, a nonzero idempotent $f$ in $e R \cap a_{i}^{\perp}$ such that $f$ is not in $a_{j}^{\perp}$. One of $f R$ or $(e-f) R$ maps onto infinitely many $a_{k} R, k \in I$. Denote the one that maps onto infinitely many $a_{k} R^{\prime}$ by $g_{1} R$, and the other one by $f_{1} R$.

Now $e R=f_{1} R \oplus g_{1} R$. Since $f \notin a_{j}^{\perp}$ and $e-f$ is not in $a_{i}^{\perp}$, it follows that $f_{1} R$ has a nonzero simple image in $B$. As $R$ is quasi-continuous and $f_{1} R \cap B=0,\left(1-f_{1}\right) R f_{1} \neq 0$. Now, since $g_{1} R$ has infinitely many simple images in $B$, repeating the same process we get idempotents $f_{2}$ and $h_{2}$ in $g_{1} R$ such that $\left(1-f_{2}\right) R f_{2} \neq 0$ and $h_{2} R$ has infinitely many simple images in $B$. Now $e R=\left(f_{1} R \oplus f_{2} R\right) \oplus h_{2} R$. Continuing this process, we get an infinite family $\left\{f_{n}: n \in N\right\}$ of orthogonal idempotents in $R$ such that $\left(1-f_{n}\right) R f_{n} \neq 0$, a contradiction to Proposition 2.8. Hence the index set $I$ is finite.

Theorem 3.6. A continuous, indecomposable, non-local $\pi$-ring $R$ has finitely generated essential socle.

Proof. In view of Theorem 2.6, we can assume that $R$ is square free. By Theorem 3.3, $\operatorname{Soc}(R) \subset^{\prime} R$. Suppose $\operatorname{Soc}(R)$ is not finitely generated. Let $\left\{S_{i}: i \in I\right\}$ be the infinite family of minimal right ideals in $R$. As $R$ is square free, this family is independent. For each $i \in I$, let $e_{i} R$ be a closure of $S_{i}$, where $e_{i}^{2}=e_{i} \in R$. By Proposition 2.8 , there are only finitely many simple $S_{i_{1}}, \ldots, S_{i_{n}}$ in $R$ such that $\left(1-e_{i_{k}}\right) R e_{i_{k}} \neq 0$, $k=1, \ldots, n$. We can pick an idempotent $e \in R$ such that both $e R$ and $(1-e) R$ contain infinitely many minimal right ideals of $R$.

By Theorem 3.5, both $e R$ and $(1-e) R$ have only finitely many simple images in each other. Of these simple images, consider only those that are not $S_{i_{k}}$ for $k=1, \ldots, n$. Now, take the closures $f R$ and $g R$ of these simple images of $e R$ and $(1-e) R$ in $(1-e) R$ and $e R$ respectively, where $f^{2}=f \in(1-e) R$ and $g^{2}=g \in e R$. Since $R$ is quasi-continuous, there exist primitive orthogonal idempotents $f_{1}, \ldots, f_{1}$, $g_{1} \ldots, g_{m}$ such that $f R=\oplus_{i=1}^{l} f_{i} R$ and $g R=\oplus_{i=1}^{m} g_{i} R$. Then $f R$ and $g R$ do not map outside themselves. Now there exist idempotents $f^{\prime} \in(1-e) R$ and $g^{\prime} \in e R$ such that $R=\left(g R \oplus g^{\prime} R\right) \oplus\left(f R \oplus f^{\prime} R\right)=\left(g R \oplus f^{\prime} R\right) \oplus\left(f R \oplus g^{\prime} R\right)$. Since $R$ is a $\pi$-ring and any nonzero image of $(1-e) R$ in $e R$ lie inside $g R$, any nonzero image of $f^{\prime} R$ in $e R$ must lie inside $g R$. If there is a nonzero homomorphism from $f^{\prime} R$ into $f R$, composing with a projection map we'll get a nonzero homomorphism from $f^{\prime} R$ onto a simple right module $S$ in $f R$. But every simple in $f R$ is an image of $e R$. As $e R$ is projective, $S$ would be isomorphic to a simple in $f^{\prime} R$. As $R$ is square free, this is a contradiction. Hence there is no nonzero homomorphism from $g R \oplus f^{\prime} R$ into $f R \oplus g^{\prime} R$. Symmetrically, there is no nonzero homomorphism from $f R \oplus g^{\prime} R$ into $g R \oplus f^{\prime} R$. But, as $R$ is 
indecomposable and $f^{\prime} R$ and $g^{\prime} R$ have infinitely many minimal right ideals, this is a contradiction. Thus, $R$ must have finitely generated essential socle.

Proposition 3.7. Let $R$ be an indecomposable, right continuous, right square free ring. Then $R$ is a right f $\pi$-ring with finite uniform right dimension if and only if $R$ is a right artinian right $\pi$-ring.

Proof. Let $R$ be an $f \pi$-ring with finite uniform dimension. Then it contains an independent family of uniform right ideals $U_{1}, \ldots, U_{n}$ with $\oplus_{i=1}^{n} U_{i} \subset^{\prime} R$. As $R$ is quasi-continuous, there exist nonzero idempotents $e_{1}, \ldots, e_{n}$ in $R$ such that $U_{i} \subset^{\prime} e_{i} R$ and $R=\oplus_{i=1}^{n} e_{i} R$. Since each $U_{i}$ is uniform it follows that each $e_{i} R$ is primitive. As $R$ is indecomposable and continuous it follows, by Lemma 3.1 that $e_{i} R\left(1-e_{i}\right) \neq 0$. By Lemma 3.2, each $e_{i} R$ is artinian. Hence $R$ is artinian. Now, since $R$ is an $f \pi$-ring, it follows that $R$ is a $\pi$-ring.

Conversely, assume that $R$ is a right artinian $\pi$-ring. If $R$ is local, then it is uniform (Lemma 1.11) and, therefore, has finite uniform dimension. If $R$ is non-local, then it has finite uniform dimension by Theorem 3.6. Hence the proof follows.

The right handed version of Theorem 3 in [4] states that a ring $R$ is a non-local indecomposable right $q$-ring containing no minimal injective right ideals if and only if $R$ is isomorphic to a ring of $n \times n$ matrices of the form

$$
M_{n}(D, V)=\left(\begin{array}{cccccc}
D & V & & & & \\
& D & V & & \\
& & D & V & \\
& & \cdot & \cdot & & \\
& & & \cdot & \cdot & V \\
V & & & & & D
\end{array}\right)
$$

with $D$ a division ring, $V$ a null algebra over $D$ with $\operatorname{dim}_{D} V=\operatorname{dim} V_{D}=1$ and $n \geq 2$. We will consider next a larger family of rings. Let $n \geq 2$ be a natural number. For $i \in\{1, \ldots, n\}$, let $D_{i}$ be a division ring. For $i \in\{1, \ldots, n-1\}$, let ${ }_{D_{i}} V_{i, i+1_{D_{i+1}}}$ be a bimodule and let $D_{n} V_{n 1_{D_{1}}}$ be a bimodule. For convenience, we will consider here, when dealing with the subscripts, addition modulo $n$ on the set $\{1, \ldots, n\}$ rather than on $\{0, \ldots n-1\}$ as is customary. We do this since the rows and columns of matrices are usually labeled by the first set and not the second. So, in particular, $n+1=1$ and therefore it suffices to say that ${ }_{D_{i}} V_{i, i+1_{D_{i+1}}}$ is a bimodule for $i=1, \ldots, n$. By $M=M_{n}\left(D_{1}, \ldots, D_{n} ; V_{12}, \ldots, V_{n-1, n}, V_{n 1}\right)$ we denote the set of $n \times n$ matrices with $(i, i)$ entry from $D_{1},(i, i+1)$ entry from $V_{i, i+1}(i=1 \ldots n)$, and all other entries zero. It is straight forward to see that $M$ is a ring under the usual matrix addition and multiplication if one assumes that $V_{i, i+1} V_{i+1, i+2}=0$ for $i=1, \ldots, n$.

In the following theorem we show that, under certain conditions, right $\pi$-rings are precisely those rings of the form

$$
M_{n}\left(D_{1}, \ldots, D_{n} ; V_{12}, \ldots, V_{n-1, n}, V_{n 1}\right)=\left(\begin{array}{cccccc}
D_{1} & V_{12} & & & & \\
& D_{2} & V_{23} & & & \\
& & D_{3} & V_{34} & & \\
& & \cdot & \cdot & & \\
& & & \cdot & \cdot & V_{n-1, n} \\
V_{n 1} & & & & & D_{n}
\end{array}\right)
$$


for which $\operatorname{dim}\left(V_{i, i+1_{D_{i+1}}}\right)=1$.

TheOREM 3.8. Let $R$ be an indecomposable, non-local ring. Then the following conditions are equivalent:

(1) $R$ is right continuous and a right $\pi$-ring.

(2) Every right ideal in $R$ is right continuous.

(3) Either $R$ is simple artinian or $R$ is right continuous, square free and there exist orthogonal primitive idempotents $e_{1}, \ldots e_{n}$ in $R$ such that $e_{i} R e_{j} \neq 0$ if and only if either $i=j$ or $j-1(\bmod n)$, each $e_{i} R$ has length two and $R=\bigoplus_{i=1}^{n} e_{i} R$.

(4) $R$ is either simple artinian or isomorphic to a ring of the form

$$
M=M_{n}\left(D_{1}, \ldots, D_{n} ; V_{12}, \ldots V_{n-1, n}, V_{n 1}\right)
$$

for some natural number $n$ and with entries such that $\operatorname{dim}\left(V_{i, i+1_{D_{i+1}}}\right)=1$.

(5) $R$ is right continuous and every right ideal in $R$ containing the $\operatorname{Soc}(R)$ is twosided.

(6) $R$ is right continuous and every essential right ideal in $R$ is two-sided.

Proof. The implications (2) $\Rightarrow(1)$ and $(5) \Rightarrow(6)$ are trivial, and (6) $\Rightarrow$ (1) following from Proposition 2.1. It remains only to prove the following implications: $(1) \Rightarrow(3) \Rightarrow(4) \Rightarrow(5)$ and $(1) \Rightarrow(2)$. Suppose (1) holds. If $R$ contains a square then $R$ is simple artinian, by Corollary 2.7. Suppose $R$ is square free. By Theorem 3.6, $R$ has finitely generated nonzero essential socle. Let $S_{1}, \ldots S_{n}$ be the minimal right ideals in $R$. Let $I=\{1, \ldots, n\}$. For each $i \in I$, let $S_{i} \subset^{\prime} e_{i} R$, where $e_{i}$ is a primitive idempotent in $R$. Clearly, $R=\oplus_{i=1}^{n} e_{i} R$. As $R$ is indecomposable, $e_{i} R\left(1-e_{i}\right) \neq 0$ for each $i \in I$ (Lemma 3.1). Hence there exists some $j \neq i, j \in I$ such that $e_{i} R e_{j} \neq 0$. As $R$ is square free and $e_{j} R$ is projective, $e_{i} R e_{k}=0$ for all $k \neq i, j, k \in I$. Since $R$ is indecomposable, there must exist some $k \in I, k \neq i$ (unless $n=2$ ) such that $e_{j} R e_{k} \neq 0$. Hence, there exists a permutation $\phi$ on $I=\{1, \ldots, n\}$ such that $e_{i} R e_{\phi(i)} \neq 0$ and $\phi(i) \neq i$ for all $i \in I$. Write $\phi=\phi_{1} \phi_{2} \ldots \phi_{k}$, a composition of disjoint cycles. Since $R$ is indecomposable, $k=1$ and $\phi$ is a cycle. Renumbering if necessary, we can write $\phi^{i}(1)=i+1$, for $i=1, \ldots, n-1$, and $\phi^{n}(1)=1$. Therefore, for each $i \in I$, we may consider the sequence of homomorphisms $\alpha_{i+1}: e_{i+1} R \rightarrow e_{i} R$ as follows: $e_{n} R \rightarrow e_{n-1} R \rightarrow \ldots \rightarrow e_{2} R \rightarrow e_{1} R \rightarrow e_{n} R$. Now, for each $i \in\{1, \ldots, n-1\}$, $\operatorname{Im} \alpha_{i+1}=S_{i}$ is simple. Moreover, for each $i<j, i, j \in\{1, \ldots n\}, e_{i} R e_{j} \neq 0$ iff $i=j$ or $i=j-1(\bmod n)$. By Lemma 3.2, each $e_{i} R$ has length two. Hence we have proved $(1) \Rightarrow(3)$. Now, assume (3) holds. If $R$ is not simple artinian then, by (3), $R$ is right continuous and there exist orthogonal primitive idempotents $e_{1}, \ldots e_{n}$ in $R$ such that $e_{i} R e_{j} \neq 0$ if and only if either $i=j$ or $i=j(\bmod n)$, each $e_{i} R$ has length two and $R=\oplus_{i=1}^{n} e_{i} R$. By Lemma 3.2, $e_{i} R e_{i}$ is a division ring for all $i \in\{1, \ldots n\}$. By Lemma 3.2, $S_{i}=e_{i} R e_{i+1}$. Thus $S_{i}$ can be viewed as a left vector space over $e_{i} R e_{i}$ and a right vector space over $e_{i+1} R e_{i+1}$. Denote each division ring $e_{i} R e_{i}$ by $D_{i}$ and each vector space $e_{i} R e_{i+1}$ by $V_{i, i+1}$. We will show that $\operatorname{dim}\left(V_{i, i+1_{D_{i+1}}}\right)=1$. Let $e_{i i}$ be the unit matrix in $M$ whose only nonzero entry is the $(i, i)$ entry and equals 1 . Then $e_{i i} M=\left(0 \ldots 0 D_{i} V_{i, i+1} 0 \ldots 0\right) \cong e_{i} R$. It is easy to check that the proper $M$-submodules of $e_{i i} M$ are precisely $\left(0 \ldots 0 W_{i, i+1} 0 \ldots 0\right)$ where $W_{i, i+1}$ is a right $D_{i+1}$-subspace of $V_{i, i+1}$. Therefore, it is clear that the $e_{i i} M$ has no non-trivial summands. Since $e_{i i} M$, being isomorphic to $e_{i} R$, is quasi-continuous it follows that it is uniform as a right $M$-module. Thus, $V_{i, i+1}$ is uniform as a right $D_{i+1}$-module. Hence, 
$\operatorname{dim}\left(V_{i, i+1_{D_{i+1}}}\right)=1$. Finally by defining $V_{i, i+1} V_{i+1, i+2}=0, i \in I$, we get the following matrix representation of $R$ :

$$
\begin{aligned}
& R=\oplus_{i=1}^{n} e_{i} R \cong\left(\begin{array}{cccccc}
D_{1} & V_{12} & & & & \\
& D_{2} & V_{23} & & \\
& & D_{3} & V_{34} & \\
& & \cdot & \cdot & & \\
& & & \cdot & \cdot & V_{n-1, n} \\
V_{n 1} & & & & & D_{n}
\end{array}\right) \\
& =M_{n}\left(D_{1}, \ldots D_{n} ; V_{12}, \ldots, V_{n-1, n}, V_{n 1}\right) \text {. }
\end{aligned}
$$

This proves (4).

Now, assume (4) holds. If $R$ is simple artinian then (5) holds trivially. Assume $R$ is square free and isomorphic to the matrix ring $M$. It is easy to check that the only proper $M$-submodules of $e_{i i} M$ are $\left(0 \ldots 0 W_{i, i+1} 0 \ldots 0\right)$, where $W_{i, i+1}$ is a right $D_{i+1^{-}}$ subspace of $V_{i, i+1}$. As $\operatorname{dim}\left(V_{i, i+1_{D_{i+1}}}\right)=1$, it is clear that $e_{i i} M$ is uniform. It is also clear that $e_{i i} M$ is not isomorphic to any of its proper submodules. It follows that each $e_{i i} M$ is continuous. It is easy to check that $\operatorname{Soc}\left(e_{i i} M\right)=\left(0 \ldots 0 V_{i, i+1} 0 \ldots 0\right)$. Therefore, as $M$ is square free, there is no nonzero homomorphism from a proper right ideal of $e_{i i} M$ to a right ideal of $e_{j j} M$ for $i \neq j$. Hence, for $i \neq j, e_{i i} M$ is $e_{j j} M$-injective. Hence $M$ is right continuous.

Now, let $a, m$ be two nonzero elements of $M$. For $i=1, \ldots, n$, there exist $d_{i}$, $\Delta_{i} \in D_{i}$ and $v_{i, i+1}, w_{i, i+1} \in V_{i, i+1}$ such that

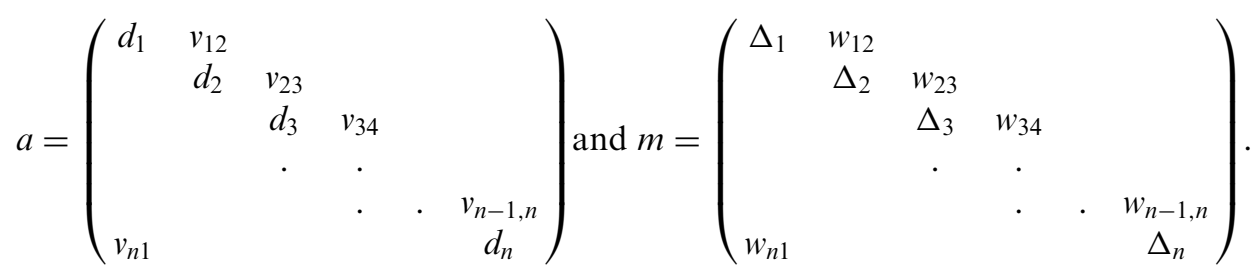

For $i=1 \ldots n-1$, define $\delta_{i}=\Delta_{i}^{-1} d_{i} \Delta_{i}$, if $\Delta_{i} \neq 0$, and $\delta_{i}=0$ otherwise. Define $u_{i, i+1}=$ $\Delta_{i}^{-1} d_{i} w_{i, i+1}$ if $\Delta_{i} \neq 0$ and $u_{i, i+1}=0$ otherwise. Define $s_{i}=v_{i, i+1} \Delta_{i+1}-w_{i, i+1} \delta_{i+1}$ if $\Delta_{i} \neq 0$ and $s_{i}=d_{i} w_{i, i+1}+v_{i, i+1} \Delta_{i+1}-w_{i, i+1} \delta_{i+1}$ if $\Delta_{i}=0$. For $i=n$, define $u_{n 1}=\Delta_{n}^{-1} d_{n} w_{n 1}$ if $\Delta_{n} \neq 0$ and $s_{n}=v_{n 1} \Delta_{1}-w_{n 1} \delta_{1}$ if $\Delta_{n} \neq 0$, and $s_{n}=v_{n 1} \Delta_{1}+$ $d_{n} w_{n 1}-w_{n 1} \delta_{1}$ if $\Delta_{n}=0$.

It is straightforward to verify that $m a=s+a m^{\prime}$, where

$$
s=\left(\begin{array}{cccccc}
0 & s_{1} & & & & \\
& 0 & s_{2} & & & \\
& & 0 & s_{3} & & \\
& & \cdot & \cdot & & \\
& & \cdot & \cdot & s_{n-1} & \\
s_{n} & & & & & 0
\end{array}\right) \text { and } m^{\prime}=\left(\begin{array}{cccccc}
\delta_{1} & u_{12} & & & & \\
& \delta_{2} & u_{23} & & & \\
& \delta_{3} & u_{34} & & \\
& \cdot & \cdot & & \\
& & \cdot & u_{n-1, n} & \\
& & & & & \delta_{n}
\end{array}\right) .
$$

Now, as $\operatorname{Soc}\left(e_{i i} M\right)=\left(0 \ldots 0 V_{i, i+1} 0 \ldots 0\right), \operatorname{Soc}(M)$ consists of all matrices in $M$ with zero diagonal. Thus, $m a \in \operatorname{Soc}(M)+a M$. Hence every right ideal of $M$ containing the $\operatorname{Soc}(M)$ is two-sided, proving (5). 
Now, suppose (1) holds. Let $I$ be a right ideal of $R$ isomorphic to a summand $e R$ of $R$ where $e^{2}=e \in R$. If $e$ is primitive then, by Lemmas 3.1 and 3.2,eR has only one proper right ideal $S=\operatorname{Soc}(e R)$. Since $e R$ is indecomposable, $I$ is indecomposable. Let $f R$ be a closure of $I$ in $R$, where $f^{2}=f \in R$. Then $f R$ is indecomposable and again, by Lemmas 3.1, 3.2, $f R$ has only one proper right ideal $T=\operatorname{Soc}(f R)$. As $e R$ is not simple, $I$ is not simple. Thus, $I=f R$. Now suppose $e$ is not primitive. As $R$ is quasi-continuous and has finite uniform dimension (Theorem 3.6), we can write $e R=f_{1} R \oplus \ldots \oplus f_{k} R$, where the $f_{i}$ are primitive idempotents. There exist submodules $I_{i}$ of $I, i=1, \ldots k$, such that $I=I_{1} \oplus \ldots \oplus I_{k}$ and $I_{i} \cong f_{i} R$. By the above arguments, each $I$, is a summand of $R$. Since $R$ is quasi-continuous, it follows that $I \subset \subset^{\oplus} R$. Hence $R$ is continuous. Thus, (2) holds.

ExAmple 3.9. In the above Theorem, the left dimension of $V_{i, i+1}$ over $D_{i}$ is not necessarily 1 , as the following example shows.

Let $F$ be any field, $F(x)$ the field of rational functions over $F$ on the variable $x$. Let $V=F(x)$ be the $F(x)$-bimodule with left action of $F(x)$ on $V$ given by $f(x) \cdot g(x)=f\left(x^{2}\right) g(x)$ and the right action given by multiplication in $F(x)$. Consider the ring

$$
R=\left(\begin{array}{ccc}
F(x) & V & 0 \\
0 & F(x) & V \\
V & 0 & F(x)
\end{array}\right)
$$

where $V^{2}=0$. This ring satisfies all the conditions in Theorem 3.6 but $\operatorname{dim}(F(x) V)=2$.

Let

$$
M^{\prime}=M_{n}^{\prime}\left(D_{1}, \ldots, D_{n} ; V_{21}, \ldots V_{n, n-1}, V_{1 n}\right)=\left(\begin{array}{ccccccc}
D_{1} & & & & & & V_{1 n} \\
V_{21} & D_{2} & & & & \\
& V_{32} & D_{3} & & & \\
& & V_{43} & \cdot & & \\
& & & \cdot & \cdot & \\
& & & & V_{n, n-1} & D_{n}
\end{array}\right) \text {, }
$$

where for $i=1 \ldots n, D_{i}$ is a division ring and $V_{i+1, i}$ is a $D_{i+1}-D_{i}$ bimodule. Notice that, as we did before, when dealing with subscripts we are considering addition modulo $n$ on the set $\{1, \ldots, n\}$ rather than on $\{0, \ldots, n-1\}$.

It is straightforward to see that $M^{\prime}$ is a ring under the usual matrix addition and multiplication if we assume that $V_{i+1, i} V_{i, i-1}=0$ for $i=1 \ldots n$.

Certainly, there is a symmetric, left-handed version of Theorem 3.8 to characterize left continuous indecomposable left $\pi$ rings in terms of rings of the form $M^{\prime}=M_{n}^{\prime}\left(D_{1}, \ldots, D_{n} ; V_{21}, \ldots V_{n, n-1}, V_{1 n}\right)$, as follows.

TheOREM 3.10. Let $R$ be an indecomposable, non-local ring. Then the following conditions are equivalent:

(1) $R$ is left continuous and a left $\pi$-ring;

(2) $R$ is either simple artinian or isomorphic to a ring of the form 


$$
M^{\prime}=M_{n}^{\prime}\left(D_{1}, \ldots, D_{n} ; V_{21}, \ldots, V_{n, n-1}, V_{1 n}\right)
$$

for some natural number $n$ and with entries such that $\operatorname{dim}\left(D_{i+1} V_{i+1, i}\right)=1$.

Proof. Similar to that of Theorem 3.8.

However, it is important to point out that one can also characterize right continuous, indecomposable, non-local rings in terms of rings of the form $M^{\prime}$, as follows.

THEOREM 3.11. Let $R$ be a right continuous, indecomposable, non-local ring. Then the following conditions are equivalent:

(1) $R$ is a right $\pi$-ring

(2) either $R$ is simple artinian or $R$ is square free and there exist orthogonal primitive idempotents $f_{1}, \ldots, f_{n}$ in $R$ such that $f_{i} R f_{j} \neq 0$ if and only if either $i=j$ or $i=j+1(\bmod n)$, each $f_{i} R$ has length two and $R=\oplus_{i=1}^{n} f_{i} R$;

(3) either $R$ is simple artinian or $R$ is square free and isomorphic to a ring of the form

$$
M^{\prime}=M_{n}\left(D_{1}, \ldots, D_{n}, V_{21} \ldots ; V_{n, n-1}, V_{1 n}\right)=\left(\begin{array}{ccccccc}
D_{1} & & & & & & V_{1 n} \\
V_{21} & D_{2} & & & & \\
& V_{32} & D_{3} & & & \\
& & V_{43} & \cdot & & \\
& & & \cdot & \cdot & & \\
& & & & V_{n, n-1} & D_{n}
\end{array}\right)
$$

for some natural number $n$ and $\operatorname{dim}\left(V_{i+1, i_{D_{i}}}\right)=1$.

Proof. Suppose (1) holds. Assume $R$ is not simple artinian. By Theorem 3.8, $R$ is right continuous, square free and there exist orthogonal primitive idempotents $e_{1}, \ldots e_{n}$ in $R$ such that $e_{i} R e_{j} \neq 0$ if and only if either $i=j$ or $i=j-1(\bmod n)$, each $e_{i} R$ has length two and $R=\oplus_{i=1}^{n} e_{i} R$. Define $f_{i}=e_{n-i+1}$. Then $f_{i} R f_{j}=$ $e_{n-i+1} R e_{n-j+1} \neq 0$ if and only if either $n-i+1=n-j+1$ or $n-i+1=n-j+1-$ $1(\bmod n)$ if and only if either $i=j$ or $i=j+1(\bmod n)$. Hence the sequence $e_{n} R \rightarrow e_{n-1} R \rightarrow \ldots \rightarrow e_{2} R \rightarrow e_{1} R \rightarrow e_{n} R$ is the same as the sequence $f_{1} R \rightarrow$ $f_{2} R \rightarrow \ldots \rightarrow f_{n} R \rightarrow f_{1} R$. This gives rise to the matrix representation $M^{\prime}$ of the ring $R$. A proof similar to the proof of Theorem 3.8 will prove the equivalence of all the statements in Theorem 3.11.

COROLlary 3.12. If $R$ is indecomposable are non-local, then the condition of being a right $q$-ring is equivalent to being a left q-ring.

Proof. A right handed version of [4, Theorem 3] states that an indecomposable, non-local right $q$-ring is either simple artinian or a ring of the form

$$
M_{n}(D, V)=\left(\begin{array}{cccccc}
D & V & & & & \\
& D & V & & \\
& & \cdot & \cdot & & \\
& & & \cdot & \cdot & V \\
V & & & & & D
\end{array}\right)
$$


where $D$ is a division ring and $V$ is a null algebra over $D$ with $\operatorname{dim}\left({ }_{D} V\right)=1=$ $\operatorname{dim}\left(V_{D}\right)$. Following the proof of Theorem 3.11, it is easy to check that an indecomposable, non-local, right $q$-ring $R$ is either simple artinian or a ring of the form

$$
M_{n}^{\prime}(D, V)=\left(\begin{array}{cccccc}
D & & & & & V \\
V & D & & & & \\
& V & D & & & \\
& & \cdot & \cdot & & \\
& & & \cdot & \cdot & \\
& & & & V & D
\end{array}\right)
$$

with $D$ and $V$ as above. By [4, Theorem 3], $M_{n}^{\prime}(D, V)$ is a left $q$-ring.

If the ring $R$ in Theorem 3.8 is both a left and right $\pi$-ring, then both the left and right dimensions of $V_{i, i+1}$, over $D_{i}$ and $D_{i+1}$ respectively, are equal to one and $R$ is a (two-sided) $q$-ring, as the following Theorem shows:

THEOREM 3.13. Let $R$ be a right continuous, indecomposable, non-local ring. Then the following conditions are equivalent:

(1) $R$ is a right and left $\pi$-ring;

(2) $R$ is a left quasi-continuous right $\pi$-ring;

(3) $R$ is either simple artinian or isomorphic to a ring of the form

$$
M=M_{n}\left(D_{1}, \ldots, D_{n} ; V_{12}, \ldots, V_{n-1, n}, V_{n 1}\right),
$$

for some natural number $n$ and with entries such that $\operatorname{dim}\left(V_{i, i+1_{D_{i+1}}}\right)=$ $1=\operatorname{dim}\left({ }_{D_{i}} V_{i, i+1}\right)$

(4) $R$ is a right q-ring;

(5) $R$ is a right and a left q-ring.

Proof. Clearly (1) implies (2). Now suppose (2) holds. Then $R$ is a right $\pi$-ring. By Theorem 3.8(4), $R$ is either simple artinian or of the form $M_{n}\left(D_{1}, \ldots D_{n}\right.$; $\left.V_{12}, \ldots, V_{n-1, n}, V_{n 1}\right)$. If $R$ is simple artinian then (3) holds trivially. Otherwise, every left summand $R e_{i} \cong\left(\begin{array}{c}0 \\ \cdot \\ \cdot \\ \cdot \\ V_{i+1, i} \\ D_{i} \\ \cdot \\ \cdot \\ \cdot \\ 0\end{array}\right)$, where $e_{i}$ is the unit matrix $e_{i i}$, has as its only 
submodules $R e_{i}$ and 'columns' of the form $\left(\begin{array}{c}0 \\ \cdot \\ \cdot \\ \cdot \\ W_{i-1, i} \\ 0 \\ \cdot \\ \cdot \\ 0\end{array}\right)$ where $W_{i-1, i}$ is a $D_{i-1}$-sub-

space of $V_{i-1, i}$. Therefore, $R e_{i}$ is indecomposable and, since it is also left quasi-continuous, the dimension of $V_{i-1, i}$ as left $D_{i-1}$-space is 1, proving (3). Now, suppose (3) holds. Let $0 \neq x \in V_{i, i+1}$. Then $V_{i, i+1}=x D_{i+1}=D_{i} x$. The assignment $d \rightarrow d^{\prime}$ if and only if $d x=x d^{\prime}$ defines a ring isomorphism between $D_{i}$ and $D_{i+1}$. Hence the $D_{i}^{\prime}$ are all isomorphic and we can view them as a division ring $D$. It is now easy to check that the $V_{i}^{\prime}$ are all isomorphic as well. Hence $R$ is a right $q$-ring [4, Theorem 3], proving (4). Now (4) $\Rightarrow(5)$ is clear by Corollary $3.12,(5) \Rightarrow(1)$ is trivial.

\section{REFERENCES}

1. C. F. Faith. Algebra I: Rings and categories of modules (Springer-Verlag, 1974). 1979).

2. K. Goodearl, Von Neumann regular rings (Pitman Publishing Limited, London,

3. K. Goodearl, Nonsingular rings and modules (Marcel Decker, New York, 1976).

4. Ivanov, Non-local rings whose ideals are all quasi-injective, Bull. Austral. Math. Soc. 45 (1972), 45-52.

5. Ivanov, Non-local rings whose ideals are all quasi-injective, Addendum, Bull. Austral. Math. Soc. 12 (1975), 159-160.

6. G. Ivanov, On a generalization of injective von-Neumann rings, to appear.

7. S. K. Jain, S. Mohamed and S. Singh, Rings in which every right ideal is quasiinjective, Pacific J. Math. 31 (1969), 73-79.

8. T. Y. Lam, A first course in noncommutative rings (Springer-Verlag, 1991).

9. S. R. López-Permouth, K. Oshiro and S. T. Rizvi, On the relative (quasi-) continuity of modules, Comm. Algebra, to appear.

10. B. J. Müller and S. Mohamed, Continuous and discrete modules (Cambridge University Press, 1990). 